\title{
Hydrodynamic and Nonhydrodynamic Contributions to the Bimolecular Collision Rates of Solute Molecules in Supercooled Bulk Water
}

\author{
Ida Peric, Dalibor Merunka, ${ }^{\dagger}$ Barney L. Bales, and Miroslav Peric* \\ Department of Physics and Astronomy and The Center for Supramolecular Studies, California State University at Northridge, \\ Northridge, California 91330, United States
}

\section{Supporting Information}

ABSTRACT: Bimolecular collision rate constants of a model solute are measured in water at $T=259-303 \mathrm{~K}$, a range encompassing both normal and supercooled water. A stable, spherical nitroxide spin probe, perdeuterated 2,2,6,6-tetramethyl-4-oxopiperidine-1-oxyl, is studied using electron paramagnetic resonance spectroscopy (EPR), taking advantage of the fact that the rotational correlation time, $\tau_{\mathrm{R}}$, the mean time between successive spin exchanges within a cage, $\tau_{\mathrm{RE}}$, and the long-time-averaged spin exchange rate constants, $K_{\mathrm{ex}}$ of the same solute molecule may be measured independently. Thus, long- and short-time translational diffusion behavior may be inferred from $K_{\mathrm{ex}}$ and $\tau_{\mathrm{RE}}$, respectively. In order to measure $K_{\mathrm{ex}}$ the effects of dipole-dipole interactions (DD) on the EPR spectra must be separated, yielding as a bonus the DD broadening rate constants that are related to the dephasing rate constant due to $\mathrm{DD}, W_{\mathrm{dd}}$. We find that both $K_{\mathrm{ex}}$ and $W_{\mathrm{dd}}$ behave hydrodynamically; that is to say

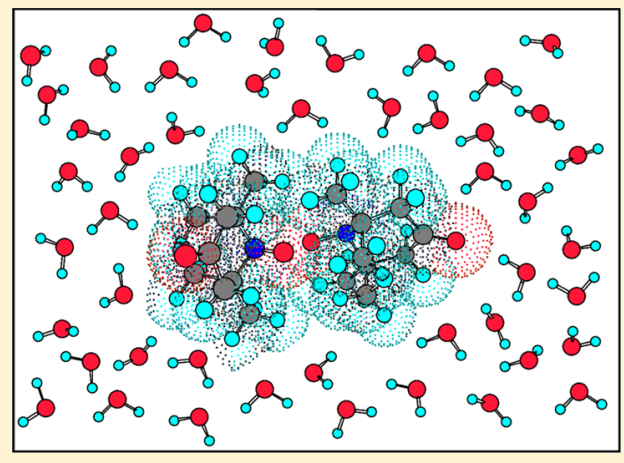
they vary monotonically with $T / \eta$ or $\eta / T$, respectively, where $\eta$ is the shear viscosity, as predicted by the Stokes-Einstein equation. The same is true of the self-diffusion of water. In contrast, $\tau_{\mathrm{RE}}$ does not follow hydrodynamic behavior, varying rather as a linear function of the density reaching a maximum at $276 \pm 2 \mathrm{~K}$ near where water displays a maximum density.

\section{INTRODUCTION}

Although ubiquitous, and one of the most studied substances on Earth, water is still shrouded in mystery. ${ }^{1-5}$ Supercooled water-water that remains liquid below its freezing pointexhibits pronounced anomalous properties, such as decreasing density, increasing isothermal compressibility, and isobaric heat capacity with decreasing temperature. ${ }^{3,6}$ Also, structural dynamics are significantly slower in supercooled water than in normal water. ${ }^{7,8}$ Recently, the rotational mobility of trehalose, a solute that strongly interacts with water through hydrogen bonding, has been studied by nuclear magnetic resonance (NMR) in aqueous solutions over a wide range of concentrations and over a temperature range encompassing the supercooled and normal regions. ${ }^{9}$ Winther et al. ${ }^{9}$ found that the trehalose tumbling rate is lower than the rate predicted by the Stokes-Einstein-Debye (SED) equation due to a secondary dynamic solvent effect, where the trehalose molecule slows down the structural dynamics of water in the hydration layer, which in turn slows down its rotation. They also found ${ }^{9}$ that the ratio between measured and limiting SED tumbling times is a nonmonotonic function of temperature showing a maximum in the supercooled region, which was explained by the fact that the activation energy for structural water dynamics changes less in the hydration layer than it does in the bulk water. An interesting question arises: does the translational diffusion of a solute follow the Stokes-Einstein relation in the supercooled region?
Rotational diffusion of solute molecules has been studied extensively through the years using electron paramagnetic resonance spectroscopy (EPR), primarily with stable nitroxide spin probes (nitroxides). In 1976, the first EPR article on supercooled water $^{10}$ presented a study of the rotational correlation time, $\tau_{\mathrm{R}}$, of the spin probe di-tert-butyl nitroxide (DTBN) in supercooled water in the temperature range 240$288 \mathrm{~K}$. The rapid increase of the ESR line widths with decreasing temperature below $273 \mathrm{~K}$ was attributed to the rapid increase of the viscosity of supercooled water. ${ }^{10}$ Another study of the rotation of DTBN in supercooled water ${ }^{11}$ reported an anomalous behavior of the hydrodynamic radius of the probe below $273 \mathrm{~K}$ that was connected to the dynamics of the clusters of water molecules with four $\mathrm{H}$-bonds and to their growth, as well as to the effect of local viscosity. Banerjee et al. ${ }^{12}$ have studied the rotation of the small polar spin probe 4-hydroxy2,2,6,6-tetramethylpiperidine-1-oxyl (TEMPOL) in interstitial deeply supercooled water of polycrystalline ice, from 90 to 300 $\mathrm{K}$. They observed two fractions of TEMPOL with different mobility and fragility in interstitial supercooled water of polycrystalline ice. By varying the degree of confinement of the supercooled water fraction in ice/water mixtures, Banerjee et al. ${ }^{13}$ have recently shown that the rotational mobility of

Received: February 6, 2014

Revised: May 28, 2014

Published: May 29, 2014 
probe molecules, surprisingly, increases in water with stronger confinement. They argued that ice-like regions are present in loose confinement, while these regions are suppressed in supercooled water with stronger confinement, which increases the fluidity of water molecules and thereby increases the rotation of the probe. Bhat et al. ${ }^{14}$ have measured the EPR spectra of TEMPOL in rapid-quench-formed amorphous water in the temperature range $140-210 \mathrm{~K}$ and have found evidence that in this region supercooled water coexists with crystalline (cubic) ice.

In nature, droplets of highly supercooled water, at temperatures as low as $235.5 \mathrm{~K}$, have been observed in deep convective clouds. ${ }^{15}$ Supercooled water at this extremely low temperature was attributed to small droplet size and the absence of ice nuclei. For that reason, the spin probe EPR method has been used to study small droplets of supercooled water confined in a polyuria microcapsule ${ }^{16}$ and a silica gel with a high hydration level. $^{17}$

Using a precise method of least-squares nonlinear EPR spectral line fitting, our group has recently studied the rotation of four small nitroxide probes in supercooled bulk water, down to $251 \mathrm{~K}^{18}$ Although the rotation of the spin probes was about an order of magnitude slower than the rotation of water molecules, when the probes' rotational correlation times were scaled to the rotational correlation time of water, their functional dependence appeared to be very similar. Interestingly, the rotational correlation times of the probes can be fit well to a power law functionality with a singular temperature of $228 \mathrm{~K}$, just like many other physical quantities. ${ }^{19}$ We also found ${ }^{18}$ that the activation energies of the rotation of the probes and water viscosity in ambient water are very close, while in the supercooled region the activation energies of the probes' rotation are greater than that of the viscosity of water. The hydrodynamic radius calculated from the rotational correlation time of the probes clearly indicated two distinct dynamical regions crossing at $277 \mathrm{~K}$. The change in hydrodynamic radius was correlated to the change in density fluctuations.

EPR has not been employed to study translational diffusion in supercooled water. In principle, Heisenberg spin exchange (HSE) studies of nitroxides provide an ideal method because of two facts: (1) spin exchange only occurs upon contact during the short period of time, $\tau_{\mathcal{c}}$ during which the unpaired spin orbitals of the two nitroxides overlap, and (2) the mean time between successive spin exchanges within a cage, $\tau_{\mathrm{RE}}$, and the long-time-averaged spin exchange rate constants, $K_{\mathrm{ex}}$ may be measured independently. ${ }^{20,21}$

The collision rate constant, $K_{\mathrm{D}}$, is related to $K_{\mathrm{ex}}$ as follows:

$$
K_{\mathrm{ex}}=\frac{1}{2} f_{\mathrm{eff}} K_{\mathrm{D}}
$$

where $f_{\text {eff }}$ is an effective steric factor. ${ }^{22}$ Therefore, a measurement of $K_{\text {ex }}$ provides an estimate of the collision rate constant. Furthermore, for nitroxides where significant spin density resides over the entire molecule, $f_{\text {eff }} \approx 1 .{ }^{22}$ The factor $1 / 2$ in eq 1 is due to the fact that only spin exchange between nitroxides with different electron spin quantum numbers affects the spectrum. $^{23}$

For identical spheres of radius $r_{\mathrm{ex}}$, the Smoluchowski equation relates $K_{\mathrm{D}}$ to the diffusion coefficient of one of the spheres, $D$, as follows:

$$
K_{\mathrm{D}}=16 \pi r_{\mathrm{ex}} D
$$

where $2 r_{e x}$ is the distance at which spin exchange occurs. ${ }^{23}$

The translational diffusion coefficient is often approximated by the Stokes-Einstein equation

$$
D=\Theta \frac{k_{\mathrm{B}} T}{6 \pi r_{\text {Stokes }} \eta}
$$

where $r_{\text {Stokes }}$ is the radius of a sphere diffusing in an incompressible fluid of shear viscosity $\eta, k_{\mathrm{B}}$ is the Boltzmann constant, and $\Theta$ is a coefficient that embodies the boundary condition (BC); $\Theta=1$ corresponds to stick and $\Theta=3 / 2$ to slip $\mathrm{BC}^{24}$ Combining eqs 2 and 3 and changing concentration units to $\mathrm{mol} / \mathrm{L}$ yield

$$
K_{\mathrm{D}}=\Theta \frac{8 R T}{3000 \eta} \cdot \frac{r_{\mathrm{ex}}}{r_{\text {Stokes }}}
$$

in units $\mathrm{L} / \mathrm{mol} \mathrm{s}$, where $R$ is the gas constant. Setting $r_{\mathrm{ex}}=r_{\text {Stokes }}$ leads to the well-known Stokes-Einstein-Smoluchowski (SES) expression, independent of the size of the sphere. ${ }^{23}$

In practice, the HSE method did not reach its full potential until recently because dipole-dipole (DD) interactions introduce spectral changes that compete with those due to HSE and a sound method to separate the two was not previously available. Recently, we have been able to separate the two effects ${ }^{25}$ by extending the theoretical approach proposed by Salikhov. ${ }^{21}$

We interpret the results of this study in terms of a simple model in which two molecules suffer a first-time collision (an encounter) with rate constant $K_{\mathrm{D}}$ followed by a series of recollisions (re-encounters) within a "cage" with mean frequency $1 / \tau_{\mathrm{RE}}$. We refer to the former as being due to macroscopic diffusion and the latter due to microscopic diffusion.

The purpose of this work is to study microscopic and macroscopic diffusion of perdeuterated 2,2,6,6-tetramethyl-4oxopiperidine-1-oxyl (pDTO) in normal and supercooled water and integrate these findings into those gleaned from studies of the rotational diffusion of the same molecule.

\section{THEORY}

Both HSE and DD broaden the lines, induce a dispersion component, and shift the lines. ${ }^{21}$ The fact that the induced dispersion and line shifts have opposite signs for DD and $\mathrm{HSE},{ }^{21,26}$ while the line broadening has the same sign, enables one to successfully separate the two contributions. ${ }^{23}$ Since the HSE-DD separation method has been published in detail in ref 25 , we will present only a short overview of it here.

The HSE rate constant may be calculated from the concentration dependence of HSE broadening, $B_{\mathrm{ex}}$, as follows

$$
K_{\mathrm{ex}}=\frac{3 \sqrt{3} \gamma}{4} \frac{\mathrm{d} B_{\mathrm{ex}}}{\mathrm{d} c}
$$

where $\gamma$ is the gyromagnetic ratio of the electron and $c(\mathrm{~mol} / \mathrm{L})$ is the concentration. Combining eqs 1,4 , and 5 yields a hydrodynamic estimate of the HSE broadening constant from the SES equation

$$
\left(\frac{\mathrm{d} B_{\mathrm{ex}}}{\mathrm{d} c}\right)_{\mathrm{SES}}=\Theta \frac{16 R f_{\text {eff }}}{9000 \sqrt{3} \gamma} \cdot \frac{r_{\mathrm{ex}}}{r_{\text {Stokes }}} \cdot \frac{T}{\eta}
$$

Using nonlinear least-squares EPR spectral fitting, ${ }^{27}$ it is possible to extract from the EPR spectrum ${ }^{28,29}$ the resonance fields of the lines, $H_{M_{I}}$, the peak-to-peak line widths $\Delta H_{\mathrm{pp}}^{0}(c)_{M_{I}}$, 
mixing parameters which are used to extract the separate Lorentzian, $\Delta H_{\mathrm{pp}}^{\mathrm{L}}(c)_{M_{\mathrm{I}}}$, and Gaussian, $\Delta H_{p p}^{G}$, contributions to $\Delta H_{\mathrm{pp}}^{0}(c)_{M_{l}}$, the peak-to-peak amplitudes of the absorption components, $\left(V_{\mathrm{pp}}\right)_{M_{I}}$, and the extremum values of the dispersion components, $\left(V_{\text {disp }}\right)_{M_{I}}$, where $M_{I}=+1,0$, and -1 represent the low-, middle-, and high-field lines, respectively. Once the spectral parameters are extracted, the total broadening of the $M_{I}$ line may be calculated as follows:

$$
B=\Delta H_{\mathrm{pp}}^{\mathrm{L}}(c)_{M_{j}}-\Delta H_{\mathrm{pp}}^{\mathrm{L}}(0)_{M_{j}}=B_{\mathrm{ex}}+B_{\text {dip }}
$$

where $B_{\text {dip }}$ is the DD broadening. $B$ is independent of $M_{I}$ for values of $B / A_{0} \ll 1$ where $A_{0}$ is the hyperfine spacing at $c=0$; however, because the outer lines broaden faster than the central line, it must be replaced by its average over three lines, $\langle B\rangle$. See Figure 7 of ref 30 .

The ratio of the dispersion extremum values to the absorption heights $\left(V_{\text {disp }}\right)_{M_{I}} /\left(V_{\mathrm{pp}}\right)_{M_{I}}$ corrected for instrumental dispersion and a small nonlinearity with $B / A_{0}$ employing eqs 3 and 4 of ref 31 denoted by $M_{I}\left(V_{\text {disp }} / V_{\mathrm{pp}}\right)_{M_{I}}^{\#}$ is proportional to $B / A_{0}$ as follows: ${ }^{25}$

$$
M_{I}\left(\frac{V_{\text {disp }}}{V_{\mathrm{pp}}}\right)_{M_{I}}^{\#}=k \frac{\langle B\rangle}{A_{0}}
$$

There are two independent values of the LHS of eq 8 which should satisfy $+\left(V_{\text {disp }} / V_{\mathrm{pp}}\right)_{+}^{\#}=-\left(V_{\text {disp }} / V_{\mathrm{pp}}\right)_{-}^{\#}$. We denote the average of the two values by $\left(V_{\text {disp }} / V_{\mathrm{pp}}\right)^{\#}$ and use one-half the difference as an estimate of the systematic fitting error. If the low-field extremum is positive and the high-field negative, HSE dominates so $k$ is positive. The opposite holds when DD dominates. $^{21,25}$

The ratio of the broadening by HSE to the overall broadening, $\Omega$, is calculated from $k$

$$
\Omega=\frac{\left\langle B_{\mathrm{ex}}\right\rangle}{\langle B\rangle}=\frac{k+2 b}{1+2 b}
$$

where $b$ depends on the details of the molecular diffusion. Using the permanent diffusion model, ${ }^{21}$ we have shown that $b$ $=4 / 19$ gives a negligible error in the separation of $\mathrm{DD}$ and HSE. $^{25}$ The values of $B_{\mathrm{ex}}$ are computed from eq 9 , and the values of $B_{\text {dip }}$, from eq 7 .

The resonance fields of the $M_{I}= \pm 1$ absorption lines are shifted by coherence transfer induced by both DD and HSE. One-half the difference in these fields, $A_{\mathrm{abs}}$, varies quadratically with $\langle B\rangle / A_{0}{ }^{23}$ Spin precession during $\tau_{\mathrm{c}}$ and during the mean time between re-encounters, $\tau_{\mathrm{RE}}$, shifts the $M_{I}= \pm 1$ lines linearly with $B_{\mathrm{ex}} / A_{0}=\Omega\langle B\rangle / A_{0} \cdot{ }^{21,25,29}$ Adding the two contributions, we get

$$
\frac{A_{\mathrm{abs}}}{A_{0}}=1-\kappa_{\mathrm{ER}} \frac{19 k+8}{27}\left(\frac{\langle B\rangle}{A_{0}}\right)-\frac{9}{32} k^{2}\left(\frac{\langle B\rangle}{A_{0}}\right)^{2}
$$

where

$$
\kappa_{\mathrm{ER}}=\frac{\sqrt{3}}{8}(1+\sqrt{2}) \sqrt{\gamma A_{0} \tau_{\mathrm{RE}} / 2}
$$

Equation 11 supposes that $\tau_{\mathrm{c}} \ll \tau_{\mathrm{RE} .}{ }^{20}$ The value of $\tau_{\mathrm{c}}$ is estimated to be approximately $10^{-13} \mathrm{~s},{ }^{22}$ while the value of $\tau_{\mathrm{RE}}$ is about $10^{-10} \mathrm{~s}$, Figure $\mathrm{S} 9$.

\section{MATERIALS AND METHODS}

Perdeuterated-Tempone (pDTO - CDN Isotopes - Lot\# P607P2) was used as received. Three stock solutions of $\mathrm{pDTO}$ at concentrations of $30.4,49.25$, and $70.39 \mathrm{mM}$ were prepared by weight in water. These solutions were diluted to other intermediate concentrations of $0.095,0.2,0.496,0.985,2.47$, $7.56,10,12.6,15,17.6,20,25.3,34.42,39.4,44.34,55.71$, 60.58 , and $65.3 \mathrm{mM}$. The samples were drawn into $5-\mu \mathrm{L}$ capillaries (radius $\approx 150 \mu \mathrm{m}$ ), which were then sealed at both ends by an open flame. EPR spectra were taken with a Bruker ESR 300E spectrometer equipped with a Bruker variable temperature unit. The sample temperature, which was held stable within $\pm 0.2{ }^{\circ} \mathrm{C}$, was measured with a thermocouple using an Omega temperature indicator. The thermocouple tip was always positioned at the top of the active region of the EPR cavity, to avoid reducing the cavity quality factor. Samples were measured in steps of $2 \mathrm{~K}$ in a temperature range from 259 to $279 \mathrm{~K}$ and in steps of $5 \mathrm{~K}$ in a temperature range from 283 to $303 \mathrm{~K}$. Samples were equilibrated at each temperature for at least $5 \mathrm{~min}$ to ensure a uniform temperature throughout the sample. Five first-harmonic EPR spectra were acquired at each temperature employing a sweep time of $84 \mathrm{~s}$, microwave power of $5 \mathrm{~mW}$, time constant of $20.5 \mathrm{~ms}$, sweep width of $50.2 \mathrm{G}$, and modulation amplitude of $0.2 \mathrm{G}$. The spectra were then transferred to a personal computer and were analyzed using the computer program Lowfit as detailed previously. ${ }^{29-31}$ After preliminary runs, the final data set includes $1590 \mathrm{EPR}$ spectra which may appear to the reader as overkill; however, our intention was to obtain very careful data with high statistics with which to test future software and theoretical ideas.

\section{RESULTS}

Figure 1 shows an experimental EPR spectrum of $70 \mathrm{mM}$ pDTO in water at $264 \mathrm{~K}$ together with its fit. To illustrate the

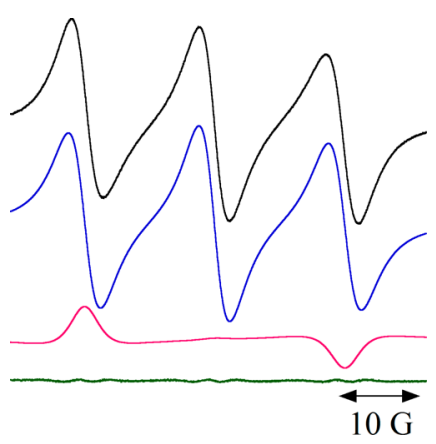

Figure 1. Experimental EPR spectrum of $70 \mathrm{mM}$ pDTO in water at $264 \mathrm{~K}$ (top trace). The second trace shows the three absorption lines, and the third, the three dispersion lines extracted from the fit of the experimental EPR spectrum. The fourth trace is the residual, the difference between the experimental spectrum, and the sum of the absorption and dispersion lines, showing that the fit is excellent; only the hyperfine lines due to ${ }^{13} \mathrm{C}$ in natural abundance are evident. Note that the positive dispersion for the low field line and negative dispersion for the high field line indicate that HSE is dominant.

EPR spectral fitting method, the experimental spectrum is separated into absorption and dispersion components. The residual, showing only minor contributions from ${ }^{13} \mathrm{C}$ lines, indicates an excellent fit. The positive values of $+\left(V_{\text {disp }} / V_{\mathrm{pp}}\right)_{+}^{\#}$ and $-\left(V_{\text {disp }} / V_{\mathrm{pp}}\right)_{-}^{\#}$ indicate that HSE dominates the DD interaction. 
The total broadening rate constant $\mathrm{d}\langle B\rangle / \mathrm{d} c$ and that due to HSE, $\mathrm{d} B_{\mathrm{ex}} / \mathrm{d} c$ as functions of $T / \eta$ for pDTO in water are presented in Figure 2. The data analysis leading to the values of

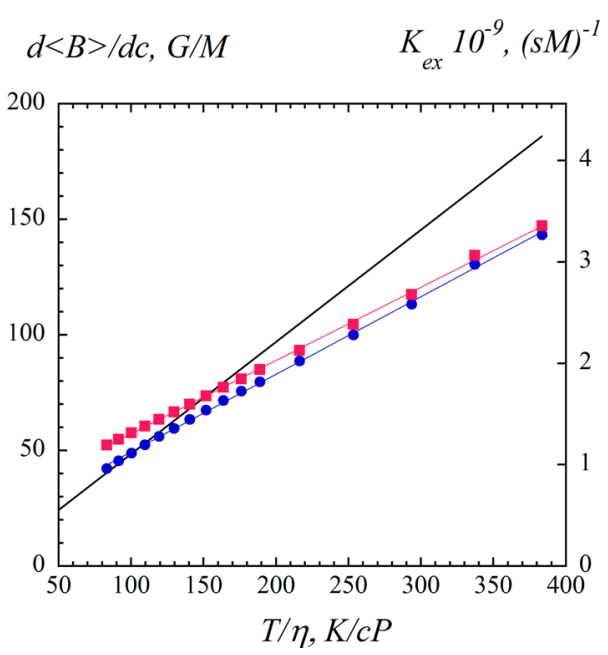

Figure 2. Total (ם) and HSE (๑) broadening constants versus $T / \eta$ for pDTO in water, left ordinate. The HSE rate constant, the right ordinate, is computed with eq 5 . The solid lines through the data are linear least-squares fits to guide the eye. The solid line is the SES prediction, eq 6. The error bars are standard deviations of five measurements and are less than the size of the symbols.

$\mathrm{d}\langle B\rangle / \mathrm{d} c$ and $d B_{\text {ex }} / \mathrm{d} c$ has been published previously ${ }^{25}$ and is reported in detail in the Supporting Information. The solid line is the SES equation, eq 6 , assuming that $r_{\text {ex }}=r_{\text {Stokes, }}$, setting $f_{\text {eff }}=$ 1 , and assuming stick $\mathrm{BC}, \Theta=1$. The right-hand ordinate, $K_{\mathrm{ex}}$ is computed from eq 5 , and $K_{\mathrm{D}}$ is twice that value assuming $f_{\text {eff }}$ $=1$.

The Stokes-Einstein (SE) expression describing the DD broadening rate constant, $\mathrm{d} B_{\mathrm{dip}} / \mathrm{d} c$, in the motional narrowing limit is as follows: ${ }^{32,33}$

$$
\left(\frac{\mathrm{d} B_{\text {dip }}}{\mathrm{d} c}\right)_{\mathrm{SE}}=C_{\mathrm{dip}} \frac{\eta}{T} \frac{r_{\text {Stokes }}}{r_{\mathrm{c}}}
$$

where $2 r_{\mathrm{c}}$ is the distance of closest approach between the two nitroxide dipoles and $\left.C_{\text {dip }}=763(\mathrm{~K} \cdot \mathrm{G}) /(\mathrm{cP} \cdot \mathrm{M})\right)^{32,33}$ The dephasing rate constant due to DD can be found from $\mathrm{d} B_{\text {dip }} / \mathrm{d} c$ using $W_{\mathrm{dd}}=(\gamma \sqrt{3} / 2) \mathrm{d} B_{\mathrm{dip}} / \mathrm{d} c{ }^{25}$ When the spin probe is immobilized, $\mathrm{d} B_{\mathrm{dip}} / \mathrm{d} c$ approaches $49.03 \mathrm{G} / \mathrm{M}^{32}$ Figure 3 shows $\mathrm{d} B_{\mathrm{dip}} / \mathrm{d} c$ and $W_{\mathrm{dd}}$ as functions of $\eta / T$. The solid line is the SE prediction, eq 12, assuming $r_{\mathrm{c}}=r_{\text {Stokes }}$. As can be observed the data are described rather well by the simple hydrodynamic prediction in that $\mathrm{d} B_{\text {dip }} / \mathrm{d} c$ varies monotonically with $\eta / T$. We note that although $\mathrm{d} B_{\text {dip }} / \mathrm{d} c$ is reasonably well described by the $\mathrm{SE}$ in water, it is very poorly described in more viscous systems. ${ }^{25,31}$

The values of $\kappa_{\mathrm{ER}}$ from the fits to eq 10 in Figure S3 can be used in eq 11 to obtain the rate of re-encounters $1 / \tau_{\mathrm{RE}}$. Figure 4 shows $1 / \tau_{\mathrm{RE}}$ versus $T / \eta$. The solid line in Figure 4 is the reciprocal of the $\mathrm{SE}$ prediction for the mean time between reencounters given by ${ }^{34}$

$$
\left(\tau_{\mathrm{RE}}\right)_{\mathrm{SE}}=\frac{3 \pi\left(2 r_{\mathrm{c}}\right)^{2} r_{\text {Stokes }} \eta}{k_{\mathrm{B}} T}
$$

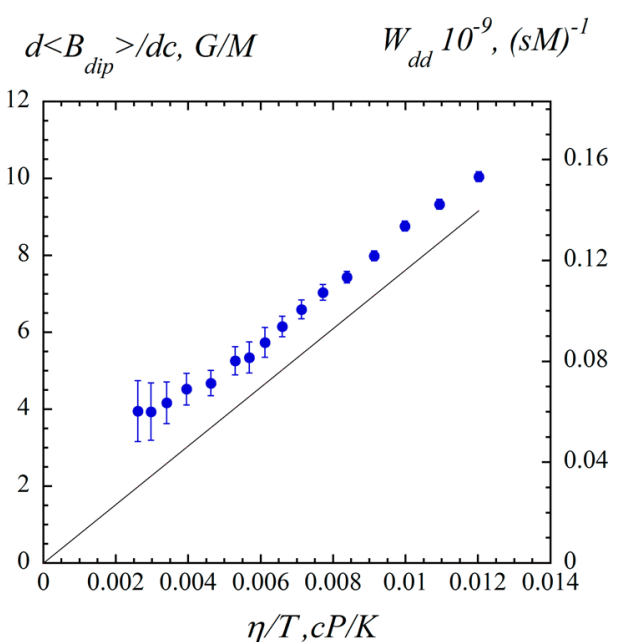

Figure 3. DD broadening constant of pDTO versus $\eta / T$ in water. The dephasing rate constant due to DD, $W_{\mathrm{dd}}=(\gamma \sqrt{3} / 2) \mathrm{d} B_{\text {dip }} / \mathrm{d} c$, the right ordinate. The solid line is the SE prediction for pDTO, eq 12. The error bars are the standard deviations of five consecutive measurements.

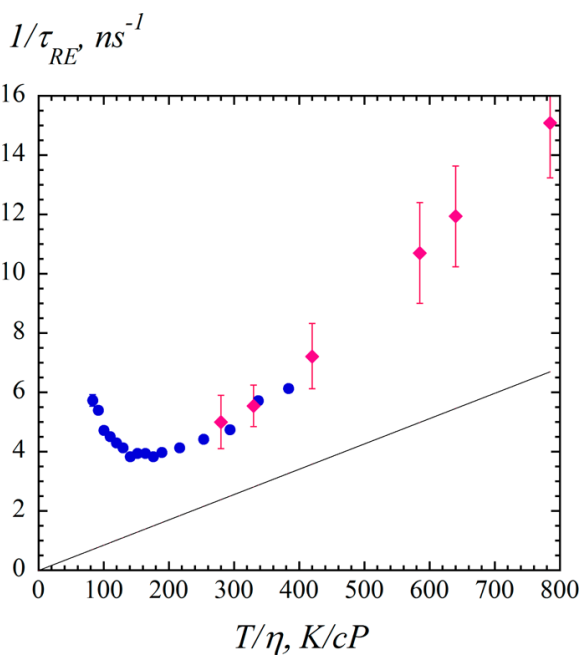

Figure 4. Re-encounter rate versus $T / \eta$ for pDTO in water. The diamonds represent the data taken from ref 49 . The solid line is the Stokes-Einstein prediction assuming $2 r_{c}=7.0 \AA$, eq 13. The error bars are the standard deviations of five consecutive measurements.

To draw the line in Figure 4, we have assumed that $r_{\text {Stokes }}$ and $r_{\mathrm{c}}$ are equal to the van der Waals radius of pDTO, $r_{\mathrm{vdW}}=3.5$ $\AA^{34}$ The behavior of $1 / \tau_{\mathrm{RE}}$ is decidedly not hydrodynamic.

\section{DISCUSSION}

From Figure 2 it can be seen that the HSE rate constant $\mathrm{d} B_{\text {ex }} / \mathrm{d} c$ of the probe behaves hydrodynamically; that is to say, it varies monotonically with $T / \eta$ and even in remarkable agreement with the SES equation, eq 6, in view of the assumptions inherent in the SES relation. Figure 3 shows that $\mathrm{d} B_{\mathrm{dip}} / \mathrm{d} c$ behaves hydrodynamically as well. These results might be expected since the $r_{\mathrm{vdW}}=3.5 \AA$ is greater than that of the water molecule, $1.4 \AA{ }^{13}$ the probe perceives the collective behavior of the surrounding water molecules as a continuum. Also, $\mathrm{d} B_{\text {ex }} / \mathrm{d} c$ and $\mathrm{d} B_{\text {dip }} / \mathrm{d} c$ do not show any noticeable difference between the supercooled and normal regions. From the point of view of macroscopic diffusion as reported 
by HSE and DD, the water in the measured temperature interval can be viewed as one kind of liquid.

Figure 4 shows a peculiar upturn in the re-encounter frequency as the temperature is decreased below $276 \pm 2 \mathrm{~K}$. Such an upturn cannot be reconciled by a hydrodynamic theory. Winther et al. ${ }^{9}$ have found that the ratio of the measured tumbling time to the limiting tumbling time, calculated from the SED equation and assuming the bulk water viscosity and hydration volume equal to the apparent solute volume as a function of temperature, is nonmonotonic and reaches a maximum in the supercooled region, at $255 \mathrm{~K}$, Figure 9 in ref 9. This maximum represents a maximal slowing of the solute due to the secondary dynamic effect of water molecules in the hydration layer. If $\tau_{\mathrm{RE}}$ is plotted as a function of $T$, Figure $S 9$ in the Supporting Information, it has a maximum just like $\tau_{\mathrm{R}} / \tau_{\mathrm{R}}^{0}$ in Figure 9 in ref 9 . This similarity might indicate that the slowing down of $\tau_{\mathrm{RE}}$ is caused by the similar interactions between pDTO and its hydration layer. On the other hand, there are several differences between these two cases. Trehalose has eight hydroxyl groups which can form hydrogen bonds, while pDTO is a nitroxide radical which can form two $\mathrm{H}$-bonds ${ }^{35}$ due to the $\mathrm{NO}^{\bullet}$ moiety, while the rest of the molecule is hydrophobic. pDTO is soluble in both water and alkanes, ${ }^{34}$ while trehalose is not soluble in alkanes. ${ }^{9}$ Also, the rotation of trehalose at $293 \mathrm{~K}, \tau_{\mathrm{R}}=117 \mathrm{ps},{ }^{9}$ is about 1 order of magnitude slower than the rotation of $\mathrm{pDTO}$ at the same temperature $\tau_{\mathrm{R}}=19 \mathrm{ps}^{18}$ Therefore, it is very likely that trehalose and pDTO interact with the hydration layer slightly differently. This can be seen by comparing the hydrodynamic radius of $\mathrm{pDTO}$ calculated by the SED equation from the measured rotational correlation time, Figure $4 \mathrm{~b}$ in ref 18 , to $\tau_{\mathrm{R}} /$ $\tau_{\mathrm{R}}^{0}$ in Figure 9 in ref 9, which shows opposite behavior. Note that the ordinates in the two figures differ just by a constant. Also, the minimum in Figure $4 \mathrm{~b}$ in ref 18 is at about $277 \pm 2 \mathrm{~K}$, which is about $20 \mathrm{~K}$ higher than the maximum in Figure 9 in ref 9.

Can the upturn in the re-encounter frequency be reconciled by turning to a free volume view of diffusion? In contrast with classical hydrodynamics, which assumes a continuous liquid, theories are based on intermolecular cavities dating from the early work of Frenkel, ${ }^{36}$ who showed that, even under full compression, free volume exists. The viscosity and diffusion are related to the free volume, thus offering an alternative view of diffusion often accompanied by ideas of "jumps." To put values of $1 / \tau_{\mathrm{RE}}$ into the context of free volume, we define a simplified geometrical fraction of free volume as follows:

$$
\varphi=\left(V-V_{\text {molecule }}\right) / V
$$

where $V$ is the volume of the sample and $V_{\text {molecule }}$ is the volume of molecules within the sample. Taking the molecules as spheres of radius $r_{\mathrm{vdW}}=1.4 \AA \mathrm{A}^{13}$ we have

$$
\varphi=1-0.38 \rho
$$

Figure 5 shows the values of $1 / \tau_{\mathrm{RE}}$ as a function of the density (bottom axis) and simplified geometrical fraction of free volume (top axis). The re-encounter rate appears to be a linear function of both density and free volume; the minimum reencounter rate occurs at $\rho=1.0 \mathrm{~g} / \mathrm{cm}^{3}$ and $\varphi=0.62$, or $277 \mathrm{~K}$. This may mean that this short-time diffusion behavior is influenced by the availability of free space into which the probe might be trapped. As the equilibrium freezing point and the temperature of maximum density have no special significance for the dynamic behavior of supercooled water, it is puzzling

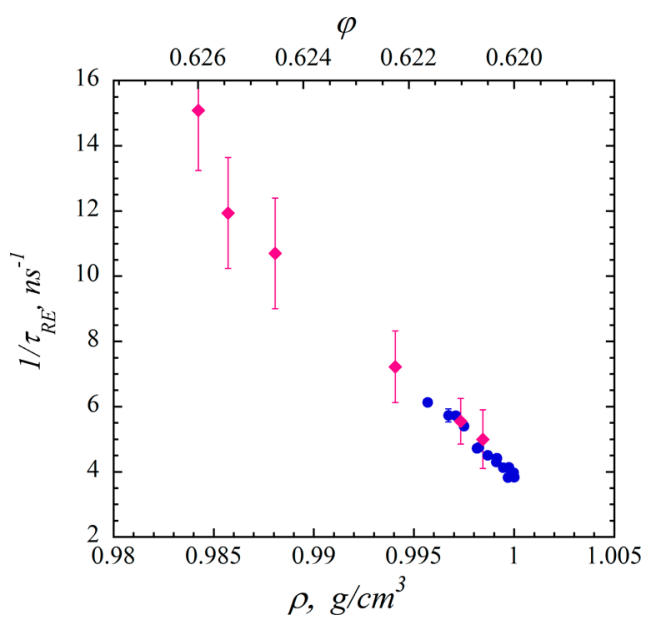

Figure 5. Re-encounter rate versus the density (bottom axis) and simplified fraction of free volume (top axis) for pDTO in water, eq 15. The diamonds represent the data taken from ref 49 . The error bars are the standard deviations of five consecutive measurements. The error bars are the standard deviations of five consecutive measurements.

that we observe the minimum re-encounter rate close to $277 \mathrm{~K}$. At the moment, we do not know why that is so; we hope that $\mathrm{MD}$ simulations of $\mathrm{pDTO}$ in water might give some answers.

Although the fractional free volume in water is high, it has been discovered that the cavities in water are distributed in smaller packets due to the small size of water molecules. ${ }^{37}$ On the other side, due to the fact that the water molecules have the same number of donor and acceptor sites arranged tetrahedrally, water can form a cage around even nonpolar solutes without disrupting much of its hydrogen bonding. 38,39 Actually, the number of $\mathrm{H}$-bonds might be slightly higher, ${ }^{38}$ so the hydration layer can be viewed as an elasticated net, ${ }^{39}$ or a dynamic cage. ${ }^{38}$ Several extensive MD studies ${ }^{40-42}$ have shown that both rotational and translational diffusion of water are affected by the presence of long-lived molecular cages. Between steps of continuous diffusion water molecules spend a considerable amount of time in cages. Similarly, we hypothesize that the nitroxide probes during an encounter might be trapped in a cage. Figure 5 suggests, but does not prove, that part of the microscopic diffusion process as reflected by $1 / \tau_{\mathrm{RE}}$ depends on free volume, in other words, the availability of cages. Note that the detailed relationship between free volume and the density depends on the system, but in any case they are very likely monotonic functions of one another.

It is of interest to compare the diffusion coefficients for pDTO derived from EPR with those for water using other techniques. Diffusion coefficients may be computed from the experimental quantities as follows: $:^{32,43}$

$$
\begin{aligned}
& D_{\mathrm{HSE}}=\frac{3 \sqrt{3} \gamma}{32 \pi N_{\mathrm{A}} 10^{-3} r_{\mathrm{ex}}} \frac{\mathrm{d} B_{\mathrm{ex}}}{\mathrm{d} c} \\
& D_{\mathrm{DD}}=\frac{19 \gamma^{3} \hbar^{2} \pi N_{\mathrm{A}} 10^{-3}}{225 \sqrt{3} r_{\mathrm{c}}}\left(\frac{\mathrm{d} B_{\mathrm{dip}}}{\mathrm{d} c}\right)^{-1} \\
& D_{\tau_{\mathrm{RE}}}=2 r_{\mathrm{c}}^{2} / \tau_{\mathrm{RE}}
\end{aligned}
$$

Figure 6 compares the diffusion coefficients of $\mathrm{pDTO}$ in water (open symbols) using EPR, eqs $16-18$, with those of water (filled symbols) using NMR, ${ }^{44}$ tracer experiments, ${ }^{45}$ and quasi-elastic neutron scattering, ${ }^{7}$ scaled by $1.4 \AA / 3.5 \AA$ to put 


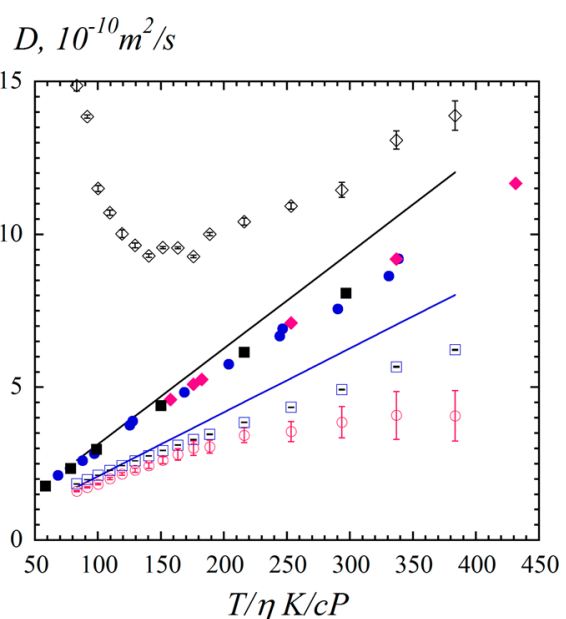

Figure 6. Translational diffusion coefficients of $\mathrm{pDTO}$ in water derived from EPR using HSE, open squares, using DD, open circles, and using re-encounter frequency, open diamonds. Translational diffusion coefficients of water scaled by $1.4 \AA / 3.5 \AA$ from $\mathrm{NMR}^{44}$ filled circles, from tracers, ${ }^{45}$ filled diamonds, and from quasi-elastic neutron scattering, ${ }^{7}$ filled squares. The upper, lower lines are the hydrodynamic predictions corresponding to slip or stick BC, respectively. These same lines correspond to the diffusion of pDTO and to the scaled values of diffusion for water.

them on the same scale of the ordinate as the diffusion coefficients of pDTO, eq 3 . The two solid lines correspond to the SES estimates using stick $\mathrm{BC}(\Theta=1$, lower line $)$ or slip $\mathrm{BC}$ $(\Theta=3 / 2$, upper line $)$, respectively. Figure 6 shows that not only pDTO but also water molecules themselves behave hydrodynamically, with water fitting numerically within the stick and slip BC of the SES relation. The data for pDTO are numerically below these limits; however, small adjustments in either $r_{\text {ex }} / r_{\text {Stokes }}$ (see ref 32 ) or $f_{\text {eff }}$ (see ref 22) could even render these data numerically consistent with the SES equation. At any rate, even without adjustments in either $r_{\text {ex }} / r_{\text {Stokes }}$ or $f_{\text {eff }}$ a hydrodynamic description is remarkably good. Clearly, translational diffusion of both water and pDTO vary smoothly through both 277 and $273 \mathrm{~K}$. Figure 6 also highlights the fact that the short-time, microscopic diffusion as reflected by $D_{\tau_{\mathrm{RE}}}$, is poorly characterized by a hydrodynamic description. Since two water molecules are hydrogen bonded to the $\mathrm{N}-\mathrm{O}^{\bullet}$ moiety, ${ }^{35}$ when two pDTO molecules encounter there could be additional hydrogen bonding between the hydrogen bonded waters of the pair. These solvent-mediated interactions might enhance the short time diffusion of pDTO above what is expected from a force-free model. Unfortunately, according to our knowledge there is no simple way to estimate the effect of these interactions. Again, perhaps MD simulations of $\mathrm{pDTO}$ in water could resolve this effect.

By using time-resolved transient absorption spectroscopy, Stickrath et al. ${ }^{46}$ measured the primary geminate recombination and cage escape times of alkyl radicals in water over a temperature range from 0 to $80{ }^{\circ} \mathrm{C}$. Caged radical pairs are produced by photodissociation from their parent molecule in a cage. Accounting for the differences between $\mathrm{pDTO}$ and the alkyl radicals, the values of $\tau_{\mathrm{RE}}$ measured in this work are several times smaller than the values of the cage escape time obtained in ref 46. In the same way, we assume that the re-encounters and rotation of pDTO very likely occur in molecular cages made of a dynamic network of tetrahedrally coordinated water molecules. ${ }^{18,40,41,46}$ Therefore, it is anticipated that these two dynamic processes should be influenced by the microstructure and microdynamics of the surrounding water molecules. From Figure 4, one can see that (i) the re-encounter rate is faster than the hydrodynamic prediction, eq 13 , assuming the distance of closest approach is two times the van der Waals radius, $b=7.0$ $\AA$, and (ii) the re-encounter rate increases as the temperature is decreased below $276 \pm 2{ }^{\circ} \mathrm{C}$. The decrease with decreasing $T / \eta$ in the normal region is as expected for a normal liquid and is the same as observed in the case of $\mathrm{pDTO}^{34}$ and the nitroxide spin probe $3 \beta$-doxyl- $5 \alpha$-cholestane in a series of $n$-alkanes. ${ }^{22}$

Recently, the rotational diffusion of $\mathrm{pDTO}$ in water was investigated over the same temperature range. There, we introduced an effective hydrodynamic radius of the probe $r_{\mathrm{R}}$ which could be found from rotational correlation time $\tau_{\mathrm{R}}$ using the Stokes-Einstein-Debye equation

$$
\tau_{\mathrm{R}}=\frac{4 \pi r_{\mathrm{R}}{ }^{3}}{3} \frac{\eta}{k T}
$$

We found that $r_{\mathrm{R}}$ varied with temperature which we attributed to the changing $\mathrm{BC}$ under rotation. The relationship between $r_{\mathrm{R}}$ and $r_{\mathrm{vdW}}$ depends on the $\mathrm{BC}$ as follows: $r_{\mathrm{R}}=0$ slip and $r_{\mathrm{R}}=r_{\mathrm{vdW}}$ for stick BC. The behavior of $r_{\mathrm{R}}$ with temperature was very similar to that of the density fluctuations, ${ }^{18}$ and it is opposite to the normalized tumbling time $\tau_{\mathrm{R}} / \tau_{\mathrm{R}}^{0}$ of trehalose ${ }^{9}$ and the time between re-encounters $\tau_{\mathrm{RE}}$ of pDTO. The similarity between Figure 4 of ref 18 and Figure 4 in this paper is that the temperature of change is the same within the experimental error, which might suggest that the $\mathrm{BC}$ under microscopic translation might be responsible for the dependence in Figure 4.

Nevertheless, we cannot conclude on the basis of the present data that a varying $\mathrm{BC}$ is responsible because the density varies in this region and secondary order dynamic solvent effects might be partially responsible. Perhaps experiments under pressure holding the density constant and $\mathrm{MD}$ simulations could resolve this issue.

\section{CONCLUSIONS}

The translational diffusion of pDTO in water and supercooled water is obtained independently from HSE and DD using our recently developed method for separating the effects of these interactions on EPR spectra. The diffusion coefficients, measured either by HSE or DD, are remarkably predicted by simple hydrodynamic considerations. The diffusion of the probe is similar to the diffusion of water when the diffusion coefficients of the two molecules are scaled according to their size. The frequency of re-encounters does not follow a hydrodynamic description, showing an upturn in frequency as the temperature is decreased below $276 \pm 2 \mathrm{~K}$. Although the reencounter time and the effective radius extracted from the rotational correlation time show opposite behavior with temperature, both of them show evident differences in normal and supercooled water. The precision of the present measurements does not permit a distinction between the melting point and the point of maximum density as the beginning of the upturn. Therefore, whether the re-encounter rate depends on the properties of the first hydration layer or on the density, or a combination of the two, cannot yet be distinguished. 


\section{ASSOCIATED CONTENT}

\section{S Supporting Information}

Text describing the method employed for data analysis. Figure S1 showing total broadening $\langle\boldsymbol{B}\rangle$ versus $\mathrm{pDTO}$ concentration in water; Figure S2 showing the values of $\left(V_{\text {disp }} / V_{\mathrm{pp}}\right)^{\#}$ versus the total normalized broadening; Figure $S 3$ showing normalized absorption hyperfine coupling spacing versus normalized broadening; Figure S4 showing an EPR spectrum for $\mathrm{pDTO}$ in the absence of HSE and DD simulated by the EasySpin ${ }^{47,48}$ function chilli; Figure S5 showing the rotational correlation time $\tau_{\mathrm{R} \text {-out }}$ extracted by spectral fitting from the EPR spectra of pDTO simulated by xchange.m (chilli) versus the rotational correlation time $\tau_{\mathrm{R}-\mathrm{in}}$; Figure S6 showing the HSE frequency $f_{\text {ex-out }}$ extracted by spectral fitting from the EPR spectra of pDTO simulated by xchange.m (chilli) versus the HSE frequency $f_{\text {ex-in; }}$ Figure $S 7$ showing normalized absorption hyperfine coupling spacing versus normalized broadening due to HSE only for different values of the rotational correlation time $\boldsymbol{\tau}_{\mathrm{R}}$ simulated by xchange.m; Figure S8 showing dispersionabsorption height ratio versus normalized broadening due to HSE only for different values of the rotational correlation time $\boldsymbol{\tau}_{\mathrm{R}}$ simulated by $x$ change. $m$; and Figure S9 showing re-encounter time and rotational correlation time versus temperature for pDTO in water. This material is available free of charge via the Internet http://pubs.acs.org.

\section{AUTHOR INFORMATION}

\section{Corresponding Author}

*E-mail: miroslav.peric@csun.edu.

\section{Present Address}

${ }^{\dagger}$ D. M. Ruder Boskovic Institute, Zagreb, Croatia.

\section{Notes}

The authors declare no competing financial interest.

\section{ACKNOWLEDGMENTS}

D.M. and M.P. gratefully acknowledge support from NIH Grant 1 SC3 GM099635-01.

\section{REFERENCES}

(1) Mishima, O.; Stanley, H. E. The Relationship between Liquid, Supercooled and Glassy Water. Nature 1998, 396, 329-335.

(2) Debenedetti, P. A. Supercooled and Glassy Water. J. Phys.: Condens. Matter 2003, 15, R1669-R1726.

(3) Debenedetti, P. A.; Stanley, H. E. Supercooled and Glassy Water. Phys. Today 2003, 56, 40-46.

(4) Angell, C. A. Insights into Phases of Liquid Water from Study of Its Unusual Glass-Forming Properties. Science 2008, 319, 582-587.

(5) Teixeira, J. Recent Experimental Aspects of the Structure and Dynamics of Liquid and Supercooled Water. Mol. Phys. 2012, 110, 249-258.

(6) Nilsson, A.; Pettersson, L. G. M. Perspective on the Structure of Liquid Water. Chem. Phys. 2011, 389, 1-34.

(7) Qvist, J.; Mattea, C.; Sunde, E. P.; Halle, B. Rotational Dynamics in Supercooled Water from Nuclear Spin Relaxation and Molecular Simulations. J. Chem. Phys. 2012, 136, 204505.

(8) Qvist, J.; Schober, H.; Halle, B. Structural Dynamics of Supercooled Water from Quasielastic Neutron Scattering and Molecular Simulations. J. Chem. Phys. 2011, 134 (144508), 1-20.

(9) Winther, L. R.; Qvist, J.; Halle, B. Hydration and Mobility of Trehalose in Aqueous Solution. J. Phys. Chem. B 2012, 116, 91969207.

(10) Ahn, M.-K. Electron Spin Relaxation of Di-tert-butyl Nitroxide in Supercooled Water. J. Chem. Phys. B 1976, 64, 134-138.
(11) Floridi, G.; Lamanna, R.; Cannistraro, S. Rotational Dynamics of Di-Tert-Butyl-Nitroxide in Normal and Supercooled Water. Appl. Magn. Reson. 1994, 7, 537-550.

(12) Banerjee, D.; Bhat, S. N.; Bhat, S. V.; Leporini, D. ESR Evidence for 2 Coexisting Liquid Phases in Deeply Supercooled Bulk Water. Proc. Natl. Acad. Sci. U.S.A. 2009, 106, 11448-11453.

(13) Banerjee, D.; Bhat, S. N.; Bhat, S. V.; Leporini, D. Molecular Probe Dynamics Reveals Suppression of Ice-Like Regions in Strongly Confined Supercooled Water. PLoS One 2012, 7, e44382(8).

(14) Bhat, S. N.; Sharma, A.; Bhat, S. V. Vitrification and Glass Transition of Water: Insights from Spin Probe ESR. Phys. Rev. Lett. 2005, 95, 235702-1-4.

(15) Rosenfeld, D.; Woodley, W. L. Deep convective clouds with sustained supercooled liquid water down to $-37.58{ }^{\circ} \mathrm{C}$. Nature 2000, 405, 440-442.

(16) Yamane, H.; Ohshima, H.; Kondo, T. Freezing Behaviour of Microencapsulated Water. J. Microencapsulation 1992, 9, 279-286.

(17) Santangelo, M. G.; Levantino, M.; Cupane, A.; Jeschke, G. Solvation of a Probe Molecule by Fluid Supercooled Water in a Hydrogel at 200 K. J. Phys. Chem. B 2008, 112, 15546-15553.

(18) Peric, I.; Merunka, D.; Bales, B. L.; Peric, M. Rotation of Four Small Nitroxide Probes in Supercooled Bulk Water. J. Phys. Chem. Lett. 2013, 508-513.

(19) Speedy, R. J.; Angell, C. A. Isothermal Compressibility of Supercooled Water and Evidence for a Thermodynamic Singularity at $-45^{\circ}$ C. J. Chem. Phys. 1976, 65, 851-858.

(20) Salikhov, K. M. The Contribution from Exchange Interaction To Line Shifts in ESR Spectra of Paramagnetic Particles in Solutions. J. Magn. Reson. 1985, 63, 271-279.

(21) Salikhov, K. M. Contributions of Exchange and Dipole-Dipole Interactions to the Shape of EPR Spectra of Free Radicals in Diluted Solutions. Appl. Magn. Reson. 2010, 38, 237-256.

(22) Vandenberg, A. D.; Bales, B. L.; Salikhov, K. M.; Peric, M. Bimolecular Encounters and Re-Encounters (Cage Effect) of a SpinLabeled Analogue of Cholestane in a Series of $n$-Alkanes: Effect of Anisotropic Exchange Integral. J. Phys. Chem. A 2012, 116 (51), 12460-12469.

(23) Molin, Y. N.; Salikhov, K. M.; Zamaraev, K. I. Spin Exchange Principles and Applications in Chemistry and Biology. Springer: Berlin, 1980.

(24) Evans, D. F.; Tominaga, T.; Davis, H. T. Tracer Diffusion in Polyatomic Liquids. J. Chem. Phys. 1981, 74, 1298-1305.

(25) Peric, M.; Bales, B. L.; Peric, M. EPR Line Shifts and Line Shape Changes Due to Heisenberg Spin Exchange and Dipole-Dipole Interactions of Nitroxide Free Radicals in Liquids: 8. Further Experimental and Theoretical Efforts to Separate the Effects of the Two Interactions. J. Phys. Chem. A 2012, 116, 2855-2866.

(26) Galeev, R. T.; Salikhov, K. M. To the Theory of Dipolar Broadening of Magnetic-Resonance Lines in Nonpolar Liquids. Chem. Phys. Reports 1996, 15, 359-375.

(27) Halpern, H. J.; Peric, M.; Yu, C.; Bales, B. L. Rapid Quantitation of Parameters from Inhomogeneously Broadened EPR Spectra. $J$. Magn. Reson. A 1993, 103, 13-22.

(28) Bales, B. L.; Peric, M. EPR Line Shifts and Line Shape Changes Due to Spin Exchange of Nitroxide Free Radicals in Liquids. J. Phys. Chem. B 1997, 101, 8707-8716.

(29) Bales, B. L.; Peric, M.; Dragutan, I. Electron Paramagnetic Resonance Line Shifts and Line Shape Changes Due to Spin Exchange between Nitroxide Free Radicals In Liquids. 3. Extension to Five Hyperfine Lines, Additional Line Shifts Due to Re-encounters. J. Phys. Chem. A 2003, 107, 9086-9098.

(30) Bales, B. L.; Peric, M. EPR Line Shifts, and Line Shape Changes Due to Spin Exchange of Nitroxide Free Radicals in Liquids 2. Extension to High Spin Exchange Frequencies and Inhomogeneously Broadened Spectra. J. Phys. Chem. A 2002, 106, 4846-4854.

(31) Bales, B. L.; Meyer, M.; Smith, S.; Peric, M. EPR Line Shifts and Line Shape Changes due to Spin Exchange of Nitroxide Free Radicals in Liquids: 6. Separating Line Broadening due to Spin Exchange and Dipolar Interactions. J. Phys. Chem. A 2009, 113, 4930-4940. 
(32) Berner, B.; Kivelson, D. The Electron Spin Resonance Line Width Method for Measuring Diffusion, A Critique. J. Phys. Chem. 1979, 83, 1406-1412.

(33) Bales, B. L.; Harris, F. L.; Peric, M.; Peric, M. Electron Paramagnetic Resonance Line Shifts and Line Shape Changes Due to Spin Exchange of Nitroxide Free Radicals in Liquids. 7. Singly Charged Surfactant Nitroxide. J. Phys. Chem. A 2009, 113, 9295-9303.

(34) Kurban, M. R.; Peric, M.; Bales, B. L. Nitroxide spin exchange due to re-encounter collisions in a series of $n$-alkanes. J. Chem. Phys. 2008, 129, 064501-10.

(35) Owenius, R.; Engström, M.; Lindgren, M.; Huber, M. Influence of Solvent Polarity and Hydrogen Bonding on the EPR Parameters of a Nitroxide Spin Label Studied ny $9-\mathrm{GHz}$ and $95-\mathrm{GHz}$ EPR Spectroscopy and DGT Calculations. J. Phys. Chem. A 2001, 105, 10967-10977.

(36) Frenkel, J. Kinetic Theory of Liquids; Dover: NY, 1955.

(37) Pohorille, A.; Pratt, L. R. Cavities in Molecular Liquids and the Theory of Hydrophobic Solubilities. J. Am. Chem. Soc. 1990, 112, 5066-5074.

(38) Galamba, N. Water's Structure around Hydrophobic Solutes and the Iceberg Model. J. Phys. Chem. B 2013, 117, 2153-2159.

(39) Schmid, R. Recent Advances in the Description of the Structure of Water, the Hydrophobic Effect, and the Like-Dissolves-Like Rule. Monatshefte für Chemie 2001, 132, 1295-1326.

(40) Sciortino, F.; Gallo, P.; Tartaglia, P.; Chen, S.-H. Supercooled water and the kinetic glass transition. Phys. Rev. E 1996, 54, 63316343.

(41) Gallo, P.; Sciortino, F.; Tartaglia, P.; Chen, S. H. Slow Dynamics of Water Molecules in Supercooled States. Phys. Rev. Lett. 1996, 76 (15), 2730-2733.

(42) Chen, S.-H.; Gallo, P.; Sciortino, F.; Tartaglia, P. MolecularDynamics Study of Incoherent Quasielastic Neutron-Scattering Spectra of Supercooled Water. Phys. Rev. E 1997, 56, 4231-4243.

(43) Abragam, A. Principles of Nuclear Magnetism; Oxford University Press: 1986.

(44) Price, W. S.; Ide, H.; Arata, Y. Self-Diffusion of Supercooled Water to $238 \mathrm{~K}$ Using PGSE NMR Diffusion Measurements. J. Phys. Chem. A 1999, 103, 448-450.

(45) Mills, R. Self-Diffusion in Normal and Heavy Water in the Range $1-45^{\circ}$. J. Phys. Chem. 1973, 77 (5), 685-688.

(46) Stickrath, A. B.; Carroll, E. C.; Dai, X.; Harris, D. A.; Rury, A.; Smith, B.; Tang, K.-C.; Wert, J.; Sension, R. J. Solvent-Dependent Cage Dynamics of Small Nonpolar Radicals: Lessons from the Photodissociation and Geminate Recombination of Alkylcobalamins. J. Phys. Chem. A 2009, 113, 8513-8522.

(47) Stoll, S.; Schweiger, A. EasySpin, a comprehensive software package for spectral simulation and analysis in EPR. J. Magn. Reson. 2006, 178 (1), 42-55.

(48) Stoll, S.; Schweiger, A. EasySpin: Simulating cw ESR spectra. Biol. Magn. Reson. 2007, 27, 299-321.

(49) Bales, B. L.; Meyer, M.; Smith, S.; Peric, M. EPR Line Shifts and Line Shape Changes Due to Spin Exchange of Nitroxide-Free Radicals in Liquids 4. Test of a Method to Measure Re-Encounter Rates in Liquids Employing ${ }^{15} \mathrm{~N}$ and ${ }^{14} \mathrm{~N}$ Nitroxide Spin Probes. J. Phys. Chem. A 2008, 112, 2177-2181. 